\title{
A Hysteresis Model for Piezoceramic Materials
}

\author{
Ralph C. Smith \\ Center for Research in Scientific Computation \\ North Carolina State University \\ Raleigh, NC 27695 \\ rsmith@eos.ncsu.edu
}

\author{
Zoubeida Ounaies \\ ICASE \\ NASA Langley Research Center \\ Hampton, VA 23681 \\ z.ounaies@larc.nasa.gov
}

\begin{abstract}
This paper addresses the modeling of nonlinear constitutive relations and hysteresis inherent to piezoceramic materials at moderate to high drive levels. Such models are necessary to realize the full potential of the materials in high performance control applications, and a necessary prerequisite is the development of techniques which permit control implementation. The approach employed here is based on the quantification of reversible and irreversible domain wall motion in response to applied electric fields. A comparison with experimental data illustrates that because the resulting ODE model is physic-based, it can be employed for both characterization and prediction of polarization levels throughout the range of actuator operation. Finally, the ODE formulation is amenable to inversion with facilitates the development of an inverse compensator for linear control design.
\end{abstract}

\section{Introduction}

Piezoceramic materials are being employed in an increasing number of structural and structural acoustic systems due to the fact that they are lightweight, compact, relatively inexpensive and provide the capability for both sensing and actuating. These advantages are bolstered by the property that they exhibit nearly linear dynamics and minimal hysteresis at low drive levels. The restriction of the materials to the quasilinear range, however, limits their output to levels which are a fraction of those attainable at moderate to high drive levels. The tradeoff for improved performance is the manifestation of the hysteresis inherent to the materials as illustrated in Figure 1 with data from a PZT 5A wafer. To utilize the full capability of piezoceramic actuators, it is necessary to model the nonlinear constitutive relations and hysteresis in a manner which is conducive to both system design and control. In this paper, we consider a model based on the domain properties of the materials.

The model is based on the theory presented in $[9,10]$ where it was illustrated for the relaxor ferroelectric material PMN-PT-BT employed in its ferroelectric regime. In light of this theory, the modeling of hysteresis in piezoceramic materials is considered in two steps. In the first, the hysteresis-free, or anhysteretic, relation between the applied field and resulting polarization is quantified through a classical application of Boltzmann statistics. This provides a constitutive relation which is applicable at low drive levels when hysteresis is negligible. Hysteresis is then modeled through the characterization of reversible and irreversible motion of domain wall pinned at inclusions in the material. The combination of the components provides an ODE model which incorporates the nonlinear constitutive relations and hysteresis observed in various PZT compounds at high drive levels.

To place this model in the context of existing hysteresis models for piezoceramic materials, we note that models can be roughly categorized as microscopic, macroscopic and semi-macroscopic in nature. The microscopic theories focus on the quantification of energy mechanisms at the lattice or domain level. As illustrated in [7], such approaches can provide a high degree of detail but are limited to simple stoichiometries 
and are currently difficult to implement in control design due to the large number of required parameters. Macroscopic theories lie at the opposite end of the spectrum and are advantageous when the underlying physical mechanisms are poorly understood or difficult to implement. This category includes models based on anhysteretic curves shifted to coincide with remanence polarizations [13] as well as the Preisach models which have been developed to quantify hysteresis in piezoceramic materials $[1,2,3]$. While advantageous in control design, these models often require a large number of nonphysical parameters which can limit their utility in systems which exhibit transient dynamics or slowly varying operating conditions. The model employed here is semi-macroscopic in the sense that it is based on energy relations for the material but employs macroscopic averages to obtain a small number (five) of effective parameters for certain material properties. Due to the physical nature of the parameters, they are easily obtained when specifying the model for particular applications and can be efficiently updated to accommodate slowly changing operating conditions.

The model is summarized in Section 2 and validated through a comparison with data from a PZT 5A wafer in Section 3. The experimental results illustrate both the accuracy of the model and its capability for predicting the polarization due to changing drive levels.

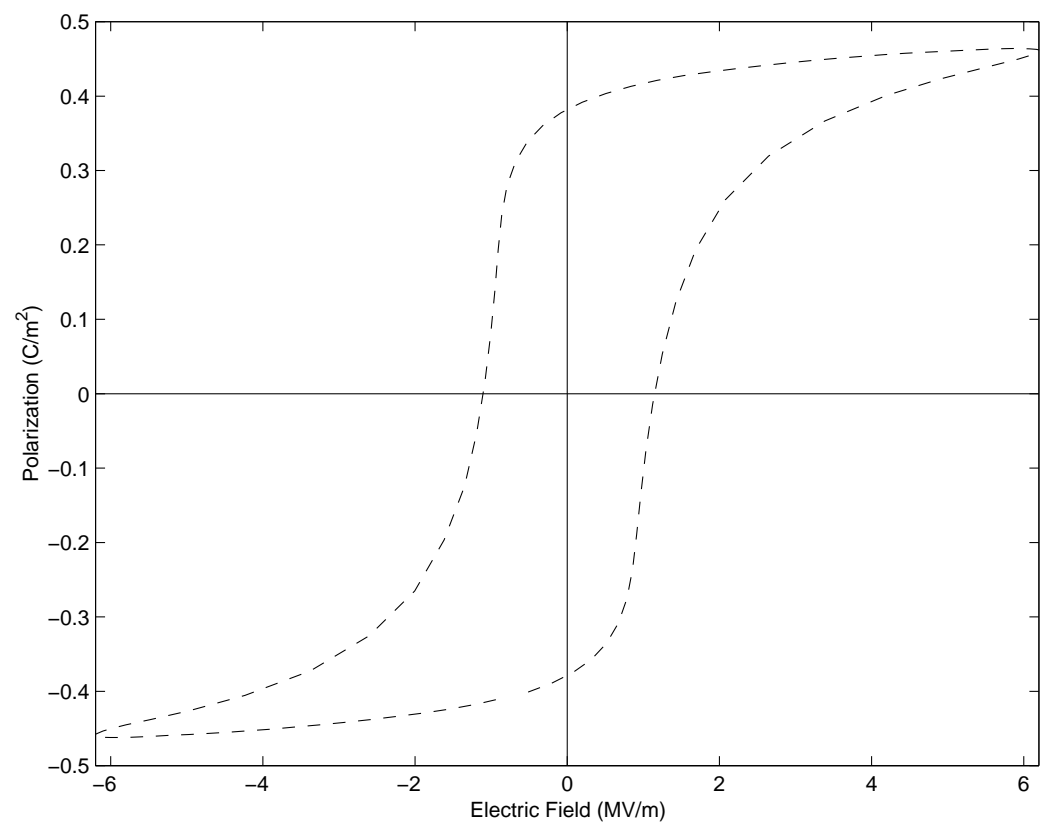

Figure 1. Hysteresis observed in a PZT 5A wafer in response to a $1600 \mathrm{~V}$ input.

\section{Hysteresis Model}

We summarize here the theory presented in $[9,10]$ for quantifying hysteresis in ferroelectric materials. This development is considered in two steps. In the first, the anhysteretic polarization is modeled through classical Boltzmann statistics. This provides the minimal energy state which would be attained by the material in the absence of pinning sites which restrict domain wall movement. Hysteresis is incorporated in the second step through the quantification of the energy required to bend and translate domain walls. This respectively provides reversible and irreversible components to the polarization which can be summed to obtain the total polarization for the material. 


\subsection{Anhysteretic Polarization}

The anhysteretic polarization curve is that which would be obtained in an idealized material which is devoid of inclusions or imperfections. As illustrated in Figure 2, the anhysteretic has a burst region near the origin, where dipole rotation toward preferred ionic configurations produces large changes in polarization, and exhibits saturation at high field levels when charge distributions prohibit further changes. In contrast to the measured polarization curves at high drive levels, the anhysteretic is single-valued and reversible.

To quantify the dependence of the anhysteretic polarization on field and coupling effects, we consider the effective field acting on moments in the material. Under the assumption of fixed temperatures, no applied stresses and quasi-static operating conditions, the effective field is taken to be

$$
E_{e}=E+\alpha P
$$

where $E$ and $P$ respectively denote the applied field and resulting polarization, and the term $\alpha P$ quantifies the field due to interdomain coupling. The parameter $\alpha=E_{0} / P_{s}$, where $E_{0}$ and $P_{s}$ respectively denote a scaling electric field and the saturation polarization, is typically estimated through a least squares fit to data. We note that throughout this development, $P_{s}$ denotes the theoretical saturation value beyond which, polar interactions prevent further increases in polarization. This definition is in accordance with the corresponding definition for the saturation magnetization (e.g., see [5]) and should not be confused with the definition employed in several texts (e.g., [4, page 36]) for the saturation value obtained by extending the slope at tip reversal back through the vertical axis.

As detailed in $[9,10]$, Boltzmann statistics is then employed to quantify the probability of dipoles occupying certain energy states. The anhysteretic models differ according to the assumptions regarding the possible dipole orientations and dielectric behavior of the material. Under the assumption that the material is isotropic and the orientation of cells can be in any direction, the balance of the thermal and electrostatic energies through a Boltzmann probability relation yields the Langevin equation

$$
P_{a n}=P_{s}\left[\operatorname{coth}\left(\frac{E_{e}}{a}\right)-\frac{a}{E_{e}}\right]
$$

for the anhysteretic polarization. The constant $a$ is specified by $a=\frac{E_{0} T}{3 T_{c}}$ where $T$ and $T_{c}$ are the operating and Curie temperatures for the material. Because the scaling field $E_{0}$ is unknown, this constant must also be estimated for a given material.

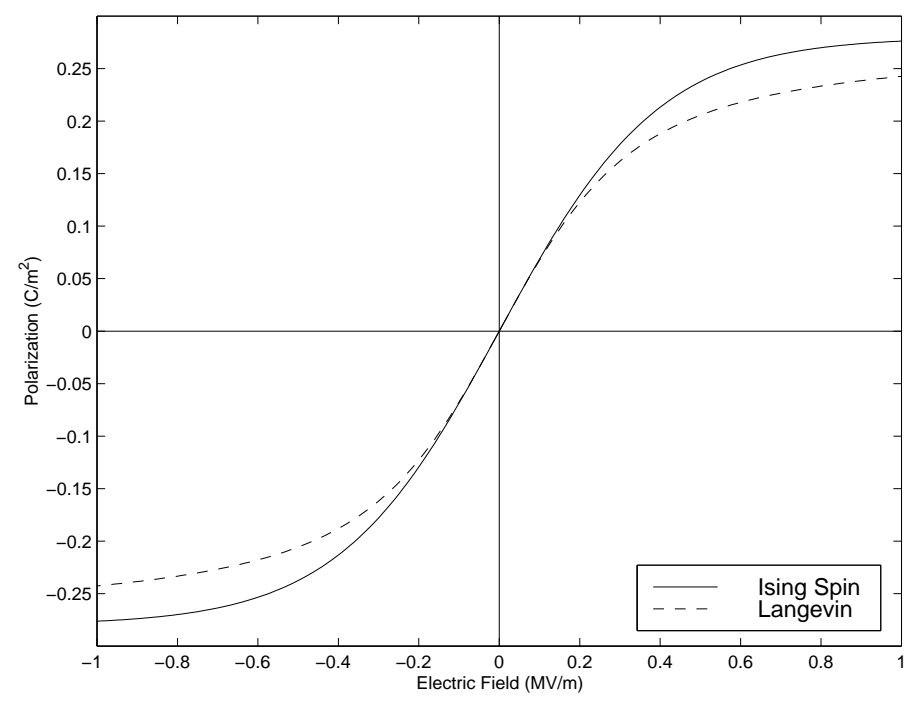

Figure 2. Ising spin and Langevin models for the anhysteretic polarization. 
A second model which is commonly employed in ferroelectric applications is the Ising spin relation

$$
P_{a n}=P_{s} \tanh \left(\frac{E_{e}}{a}\right)
$$

which results from the assumption that dipole moments can only be oriented in the direction of the field or opposite to it. As noted in Figure 2 where the two models are compared, the restrictions on possible moment orientation causes the Ising spin relation to saturate more quickly than the Langevin model. Further discussion concerning the merits of the two relations as well as details regarding their derivation are provided in $[9,10]$.

\subsection{Domain Wall Model}

The relations (2) or (3) can be employed at low drive levels but are inappropriate at moderate to high drive levels since they do not incorporate the hysteresis inherent to the materials. As detailed in [10] and included references, sigmoidal hysteresis of the type depicted in Figure 1 is typically attributed to the energy required to translate domain walls across pinning sites in the material. The pinning of domain walls by defects or inclusions in the material is due to the reduction in energy which occurs at these sites. The equilibrium position for the domain wall occurs when the potential energy is minimized. At low field levels, the walls remain close to the equilibrium position and the motion is reversible. From an energy perspective, the variations are not sufficient to cross a barrier in the potential well. The motion becomes irreversible when sufficient energy is provided to cross the potential barrier. Physically, this can occur when the domain wall intersects a remote pinning site and is the mechanism underlying domain wall translations. The resulting irreversible polarization $P_{i r r}$ and reversible polarization $P_{r e v}$ are then summed to obtain the total polarization. This approach follows that employed by Jiles and Atherton in their corresponding hysteresis model for ferromagnetic materials [6].

To quantify the irreversible polarization, it is noted in $[9,10]$ that the polarization level for a given effective field can be expressed as that for the ideal case minus losses required to break pinning sites. This yields the relation

$$
P_{i r r}=P_{a n}-\delta k \frac{d P_{i r r}}{d E_{e}}
$$

where the parameter $\delta=\operatorname{sign}(d E)$ ensures that the energy required to break pinning sites always opposes changes in polarization. The parameter $k$ is defined by $k=\frac{n\left\langle\mathcal{E}_{\pi}\right\rangle}{2 p}$ where $n$ denotes the average density of pinning sites, $\left\langle\mathcal{E}_{\pi}\right\rangle$ is the average energy for $180^{\circ}$ walls and $p$ is an average dipole moment. Because the density and energy of individual pinning sites are unknown, the parameter $k$ must be estimated for a given material.

The expansion of (4) yields the differential equation

$$
\frac{d P_{i r r}}{d E}=\frac{P_{a n}-P_{i r r}}{\delta k-\alpha\left(P_{a n}-P_{i r r}\right)}
$$

specifying the irreversible polarization. As discussed in $[9,10]$, while this expressions is adequate in most regimes, it can yield nonphysical solutions when the field is reversed from saturation for materials which exhibit significant hysteresis and are driven at high levels. The enforcement of solely reversible polarization changes in this regime eliminates this discrepancy and yields the relation

$$
\frac{d P_{i r r}}{d E}=\widetilde{\delta} \frac{P_{a n}-P_{i r r}}{k \delta-\alpha\left(P_{a n}-P_{i r r}\right)}
$$

where

$$
\widetilde{\delta}=\left\{\begin{array}{l}
1,\left\{d E>0 \text { and } P>P_{a n}\right\} \text { or }\left\{d E<0 \text { and } P<P_{a n}\right\} \\
0, \text { otherwise }
\end{array}\right.
$$


The second component of the polarization is the reversible polarization which models the effects of domain wall bending. To first approximation, this is modeled by the relation

$$
P_{\text {rev }}=c\left(P_{a n}-P_{\text {irr }}\right)
$$

where $c$ is a parameter which must be estimated for the specific application (see $[9,10])$.

The total polarization is then given by

$$
P=P_{r e v}+P_{i r r}
$$

or equivalently,

$$
P=c P_{a n}+(1-c) P_{i r r} .
$$

To implement the model, the effective field for a given field and irreversible polarization level is computed using (1). This effective field value is then employed in either (2) or (3) to compute the corresponding anhysteretic polarization. The subsequent irreversible polarization is determined by numerically integrating (5). The total polarization is then specified by (7). Various aspects of the model are illustrated in the next section through a comparison with experimental data.

\section{Model Validation}

To illustrate the performance of the model, we consider the characterization of the polarization generated by a cylindrical PZT 5A wafer. The wafer had a diameter of 1 in and was 10 mils thick. To maintain quasistatic operating conditions, the frequency of the input electric field was taken to be $200 \mathrm{mHz}$. Because the material was initially polarized prior to the hysteresis measurements, the first cycle was omitted because it exhibited transient behavior. Three complete steady state cycles were then measured for input voltages ranging from $600 \mathrm{~V}$ to $1600 \mathrm{~V}$. The corresponding field inputs to the model were determined using the relation $E=V / d$ where $d=10$ mils is the thickness of the wafer.

Two strategies were considered to illustrate the capability of the model to quantify the inherently hysteretic relation between the input field and generated polarization at various drive levels. In the first, the parameters $\alpha, a, c, k$ and $P_{s}$ were obtained through a least squares fit to polarization data collected with a peak input voltage of $600 \mathrm{~V}$. The model, with these parameters, was then used to predict the polarization at drive levels up through $1600 \mathrm{~V}$. The second option differed in that parameters were obtained by fitting the $1600 \mathrm{~V}$ data and the model was then used to predict the polarization behavior at lower drive levels.

The model behavior and corresponding data for the first strategy are illustrated in Figure 3 . The parameter values $\alpha=3.7 \times 10^{6} \mathrm{Vm} / \mathrm{C}, a=4.1 \times 10^{5} \mathrm{C} / \mathrm{m}^{2}, c=0.35, k=1.8 \times 10^{6} \mathrm{C} / \mathrm{m}^{2}$ and $P_{s}=0.49$ $\mathrm{C} / \mathrm{m}^{2}$ were obtained through a least squares fit to the $600 \mathrm{~V}$ data with the Langevin relation used to model the anhysteretic polarization. The model predictions at $800 \mathrm{~V}, 1000 \mathrm{~V}$ and $1600 \mathrm{~V}$ were then compared with the corresponding data at these drive levels. Throughout the range of drive levels, the model accurately predicts the measured polarization. We note that the capability of the model for predicting the polarization at various drive levels is due to the fact that it is based on energy principles.

The identification of parameters at $1600 \mathrm{~V}$ produced model fits that were slightly more accurate at that level but were less accurate when predicting at $600 \mathrm{~V}$. For this data set, the $600 \mathrm{~V}$ data appears to provide more information relevant to identifying the parameters and hence gives the model slightly better predictive capabilities than identification at high drive levels. For general PZT applications, however, determination of parameters and comparison of model performance at both low and high drive levels will indicate which parameters provide the best overall fit. An advantage of this approach is that once the

parameters are determined, the model can be used to quantify the polarization for a large range of drive levels simply by specifying the input voltage or field. 

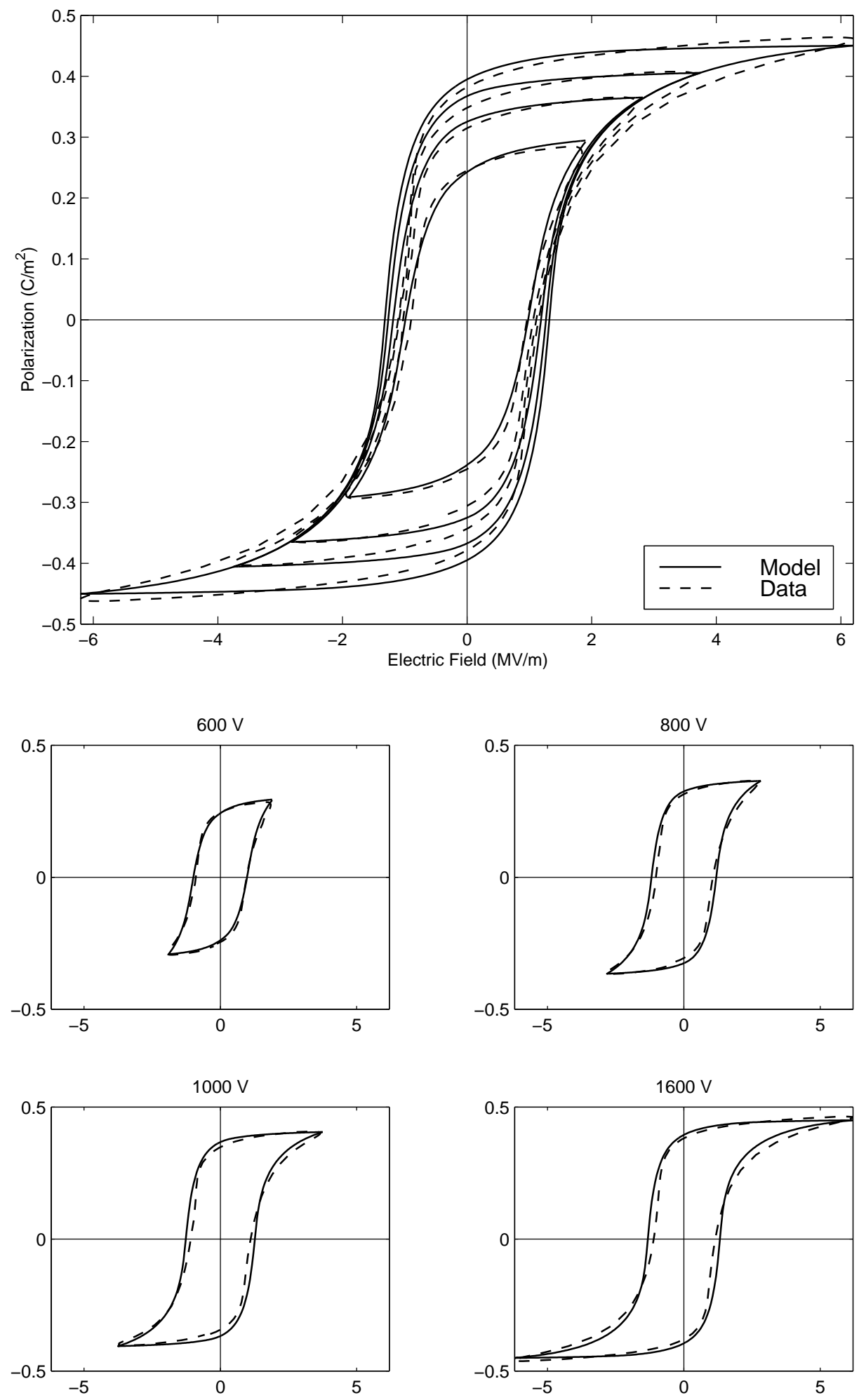

Figure 3. Model fit to $600 \mathrm{~V}$ data and model predictions at $800 \mathrm{~V}, 1000 \mathrm{~V}$ and $1600 \mathrm{~V}$ with the parameter choices $\alpha=3.7 \times 10^{6} \mathrm{Vm} / \mathrm{C}, a=4.1 \times 10^{5} \mathrm{C} / \mathrm{m}^{2}, c=0.35, k=1.8 \times 10^{6} \mathrm{C} / \mathrm{m}^{2}$ and $P_{s}=0.49 \mathrm{C} / \mathrm{m}^{2}$; Model (-), Data (-- $)$. 


\section{Concluding Remarks}

This paper summarizes a hysteresis model for piezoceramic actuators which is based on domain wall theory for ferroelectric materials $[9,10]$. The theory quantifies the hysteresis inherent to the materials through the consideration of the energy required to bend and translate domain walls which are pinned at defects or inclusions in the material. This provides reversible and irreversible polarization components whose sum represents the polarization due to an applied field. Characterization in this manner provides the model with the capability for specifying the polarization at a variety of input field levels with one set of model parameters. The flexibility of the model is further augmented by the small number (five) of required parameters and the physical nature of the parameters (e.g., $P_{s}$ is often known a priori or can be directly obtained from the data).

The performance of the model was illustrated through a comparison with data from a PZT 5A wafer. The resulting model behavior was accurate both at the drive level used to estimate the parameters as well as input fields up to more than double that magnitude. This illustrated the flexibility of the model and its capability for prediction throughout the drive range of the actuator.

Finally, the ODE nature of the model makes it amenable to inversion through the consideration of a complementary ODE in a manner analogous to that described in [8]. This facilitates the construction of an inverse compensator which can be used for linear control design [11, 12]. The application of these

techniques for linear control implementation for piezoceramic actuators which exhibit hysteresis is under investigation.

\section{Acknowledgements}

The research of R.C.S. was supported in part by the Air Force Office of Scientific Research under the grant AFOSR-F49620-98-1-0180.

\section{References}

[1] W.S. Galinaitis and R.C. Rogers, "Compensation for Hysteresis Using Bivariate Preisach Models," SPIE Smart Structures and Materials, 1997, Mathematics and Control in Smart Structures, San Diego, CA, 1997.

[2] P. Ge and M. Jouaneh, "Modeling Hysteresis in Piezoceramic Actuators," Precision Engineering, 17, pp. 211-221, 1995.

[3] P. Ge and M. Jouaneh, "Tracking Control of a Piezoceramic Actuator," IEEE Transactions on Control Systems Technology, 4(3), pp. 209-216, 1996.

[4] B. Jaffe, W.R. Cook, Jr. and H. Jaffe, Piezoelectric Ceramics, Academic Press, New York, 1971.

[5] D.C. Jiles, Introduction to Magnetism and Magnetic Materials, Chapman and Hall, New York, 1991.

[6] D.C. Jiles and D.L. Atherton, "Theory of Ferromagnetic Hysteresis," Journal of Magnetism and Magnetic Materials, 61, pp. 48-60, 1986.

[7] M. Omura, H. Adachi and Y. Ishibashi, "Simulations of Ferroelectric Characteristics Using a OneDimensional Lattice Model," Japanese Journal of Applied Physics, 30(9B), pp. 2384-2387, 1991. 
[8] R.C. Smith, "Inverse Compensation for Hysteresis in Magnetostrictive Transducers," CRSC Technical Report CRSC-TR98-36; Mathematical and Computer Modeling, to appear.

[9] R.C. Smith and C.L. Hom, "A Domain Wall Model for Ferroelectric Hysteresis," SPIE Conference on Mathematics and Control in Smart Structures, SPIE Volume 3667, Newport Beach, CA, March 1-4, 1999, pp. 150-161.

[10] R.C. Smith and C.L. Hom, "A Domain Wall Theory for Ferroelectric Hysteresis," CRSC Technical Report CRSC-TR99-1; Journal of Intelligent Material Systems and Structures, to appear.

[11] G. Tao and P.V. Kokotović, Adaptive Control of Systems with Actuator and Sensor Nonlinearities, John Wiley and Sons, New York, 1996.

[12] B. Widrow and E. Walach, Adaptive Inverse Control, Prentice Hall, NJ, 1996.

[13] X.D. Zhang and C.A. Rogers, "A Macroscopic Phenomenological Formulation for Coupled Electromechanical Effects in Piezoelectricity," Journal of Intelligent Material Systems and Structures, 4, pp. 307316, 1993. 